\title{
Differential effects and interactions of endogenous and horizontally acquired H-NS-like proteins in pathogenic Escherichia coli
}

Claudia M. Müller, ${ }^{1+}$ György Schneider, ${ }^{2,3}$

Ulrich Dobrindt, ${ }^{4}$ Levente Emödy, ${ }^{2,3}$ Jörg Hacker ${ }^{4,5}$ and Bernt Eric Uhlin ${ }^{1 *}$

${ }^{1}$ Department of Molecular Biology, The Laboratory for Molecular Infection Medicine Sweden (MIMS), Umeå University, Umeå, S-90187 Umeå, Sweden.

${ }^{2}$ Veterinary Medical Research Institute, Hungarian

Academy of Sciences, Budapest, Hungary.

${ }^{3}$ Institute of Medical Microbiology and Immunology,

University of Pécs Medical School, Pécs, H-7624 Pécs, Hungary.

${ }^{4}$ University of Würzburg, Institute for Molecular Infection Biology, Würzburg, D-97070 Würzburg, Germany.

${ }^{5}$ Robert Koch-Institut, Berlin, D-13353 Berlin, Germany.

\section{Summary}

The nucleoid-associated protein H-NS is important for gene regulation in Escherichia coli. We have studied $\mathrm{H}-\mathrm{NS}$ interaction with StpA and an uncharacterized $\mathrm{H}-\mathrm{NS}$-like protein, $\mathrm{Hfp}$, in the uropathogenic $E$. coli isolate 536 that expresses all three nucleoidassociated proteins. We found distinct interactions of the three proteins at the protein level, resulting in the formation of heteromers, as well as differences in their gene expression at the transcriptional level. Mutants lacking either StpA or Hfp alone did not exhibit a phenotype at $37^{\circ} \mathrm{C}$, which is consistent with a low level of expression at that temperature. Expression of the hfp and stpA genes was found to be induced by apparently diametrical conditions, and StpA and Hfp levels could be correlated to modulatory effects on the expression of different H-NS targets, the bgl operon and operons for virulence factors such as fimbriae and capsular polysaccharide. The $h n s / h f p$ and $h n s / s t p A$ double

Accepted 21 November, 2009. *For correspondence. E-mail bernt. eric.uhlin@molbiol.umu.se; Tel. (+46) 90785 6731; Fax (+46) 9077 $2630 .{ }^{\dagger}$ Present address: School of Biosciences, University of Exeter, Exeter EX4 4QD, UK.

Re-use of this article is permitted in accordance with the Terms and Conditions set out at http://www3.interscience.wiley.com/ authorresources/onlineopen.html mutants displayed severe growth defects at low and high temperatures respectively. Our findings demonstrated different requirements for the alternative $\mathrm{H}-\mathrm{NS} / \mathrm{Hfp} / \mathrm{StpA}$ combinations under these growth conditions. We propose that Hfp and StpA have distinct functions and roles in a dynamic pool of nucleoid-associated proteins that is adapting to requirements in a particular environment.

\section{Introduction}

The bacterial nucleoid is organized in a heterogeneous manner containing areas with highly condensed, quiescent DNA, and areas that are accessible for proteins involved in DNA processes such as replication, repair and transcription. The distribution of transcriptional activity along the chromosome is dynamic and changes rapidly according to the current requirements of the cell (Travers and Muskhelishvili, 2005). In Escherichia coli, several small proteins with DNA-binding activity play an important role in shaping the structure of the nucleoid and, simultaneously, in ensuring its flexibility. These so-called nucleoid-associated proteins affect the architecture of the nucleoid by either bending or bridging DNA (Dame, 2005; Luijsterburg et al., 2006).

One of the most abundant nucleoid-associated proteins is the histone-like nucleoid structuring protein $\mathrm{H}-\mathrm{NS}$. The protein consists of a C-terminal DNA-binding domain and an N-terminal oligomerization domain (Ueguchi et al., 1997; Renzoni et al., 2001; Bloch et al., 2003). The precise mechanism of DNA binding and nucleoprotein complex formation by $\mathrm{H}-\mathrm{NS}$ is still unclear, despite many efforts in the last years. For instance, higher-order complexes of $\mathrm{H}$-NS molecules bound along the DNA, or bridges between two adjacent DNA stretches mediated by $\mathrm{H}-\mathrm{NS}$ have been shown (Rimsky et al., 2001; Dame et al., 2005), both with potential huge impact on DNA processes. The effects of $\mathrm{H}-\mathrm{NS}$ have been studied in detail in non-pathogenic $E$. coli, where it first was suggested to cause transcriptional silencing and now is considered as a global regulator of gene expression (Göransson et al., 1990; Atlung and Ingmer, 1997; Hommais et al., 2001). In uropathogenic E. coli strain 
536, we could also demonstrate a major role of $\mathrm{H}-\mathrm{NS}$ on the expression of more than 500 genes, including many virulence factors such as fimbriae, cytotoxins and siderophores (Müller et al., 2006).

A second member of the H-NS-like protein family that is found in both pathogenic and non-pathogenic E. coli variants is the StpA protein. StpA shares $58 \%$ sequence identity to $\mathrm{H}-\mathrm{NS}$ and has a similar domain structure (Cusick and Belfort, 1998). It was shown to bind both DNA and RNA, the latter conferring RNA chaperone activity (Zhang et al., 1996; Sonnenfield et al., 2001; Mayer et al., 2007). Mutations in the $\operatorname{stp} A$ gene do not result in a notable phenotype under standard growth conditions, but StpA can compensate for the lack of $h n s$ in repression of some genes, thereby being considered as a molecular back-up for H-NS (Sondén and Uhlin, 1996; Zhang et al., 1996). Moreover, StpA is directly interacting with the H-NS protein by forming heteromers (Johansson et al., 2001).

The progress in genome sequencing led to the discovery of several genes encoding homologues or interaction partners for H-NS (Bertin et al., 2001; Tendeng and Bertin, 2003). Interestingly, many of those are encoded on transmissive genomic elements and many are also involved in virulence. One example is the plasmid-encoded $\mathrm{H}$-NS-like protein of Shigella flexneri, Sfh (Beloin et al., 2003; Doyle and Dorman, 2006). Sfh shares about 60\% amino acid homology with $\mathrm{H}-\mathrm{NS}$ and StpA and it is able to interact with both proteins (Deighan et al., 2003). Furthermore, it is able to complement an hns mutation and affects virulence gene expression when overexpressed, but also plays an additional role in transmission and maintenance of its encoding plasmid (Beloin et al., 2003; Doyle et al., 2007). Acquisition of horizontally acquired proteins that are able to interact with $\mathrm{H}-\mathrm{NS}$ might be an important step in fine tuning the pre-existing regulatory network of the host cell, which might explain the occurrence of several H-NS-like proteins within some bacterial species.

In this study, we provide evidence that the uropathogenic E. coli strain 536, but not non-pathogenic K-12 laboratory strains, harbours a third member of the H-NS-like protein family, the Hfp protein, which shares homology with both $\mathrm{H}-\mathrm{NS}$ and StpA. The gene coding for Hfp is located on a genomic island inserted at the serU locus, and its expression is repressed by H-NS. Like StpA, the Hfp protein has biological activity and can complement several hns phenotypes. To study the functions and interplay of the horizontally acquired Hfp protein and the endogenous regulators $\mathrm{H}-\mathrm{NS}$ and StpA, a series of mutant derivatives were constructed and searched for differences in their phenotype. Possible interactions between the three regulators were studied at the protein and the transcriptional level. The results obtained verified that a horizontally acquired regulator such as Hfp can be successfully integrated into the regulatory circuits of the cell. Moreover, our results from growth experiments and transcription analysis of the three corresponding genes led to the hypothesis of a flexible composition of the nucleoid-associated protein pool with changing temperature or with growth phase, resulting in distinct heteromeric protein complexes.

Results

Extraintestinal pathogenic E. coli variants commonly express three members of $\mathrm{H}$-NS-like proteins

The genes coding for $\mathrm{H}-\mathrm{NS}$ and StpA can be found in the chromosome of all characterized $E$. coli variants. In this study, the genome of uropathogenic E. coli strain 536 (Accession No. CP000247), as a newly sequenced representative of extraintestinal pathogenic E. coli (ExPEC) isolates, was searched for the presence of $\mathrm{H}$-NS-like proteins. Like E. coli K-12 strains, UPEC (uropathogenic E. coli) strain 536 possesses both the hns and the stpA equivalents. However, a third hit was obtained represented by gene ECP_1927, which is annotated as a putative DNA-binding protein (Accession No. ABG69928). The sequence encodes a protein of 134 amino acids (a.a.), which was now termed Hfp for H-NS-family protein. Hfp exhibits high homology to both H-NS and StpA (57.7\% and $52.2 \%$ a.a. identity respectively) over the entire length of the sequence (Fig. 1A). When comparing the chromosomal region in the vicinity of the hfp gene with the corresponding region in the genome of the K-12 strain MG1655, a $23 \mathrm{~kb}$ large DNA stretch including the $h f p$ gene was only present in the genome of UPEC strain 536 (Fig. 1B). The insertion was flanked by direct repeats associated with the serU tRNA locus and the overall GC content $(38 \%)$ differed from the rest of the genome (50\%), all of which are qualifiers for horizontally acquired DNA regions, so-called genomic islands.

In order to further investigate the occurrence of $\mathrm{Hfp}$ in other E. coli sero- and pathotypes, a collection of 133 isolates comprising both extraintestinal and intestinal pathogenic E. coli isolates (ExPEC and IPEC) as well as faecal isolates from healthy volunteers was screened for the presence of the hfp gene by PCR. A $355 \mathrm{bp}$ PCR product could be amplified in 55 out of the 133 strains (Table 1). Most of the hfp-positive strains belonged to the ExPEC group (58\% positive isolates), with UPECs as prevalent subgroup (79\% positive isolates). The hfp gene was also present in a high number of faecal strains (39\% positive isolates).

Taken together, these results indicate that UPEC strain 536 , and probably a majority of other uropathogenic $E$. coli isolates, carries genes for three highly homologous nucleoid-associated proteins: the core chromosomeencoded proteins H-NS and StpA, as well as the so far uncharacterized Hfp protein. 
A Oligomerization domain

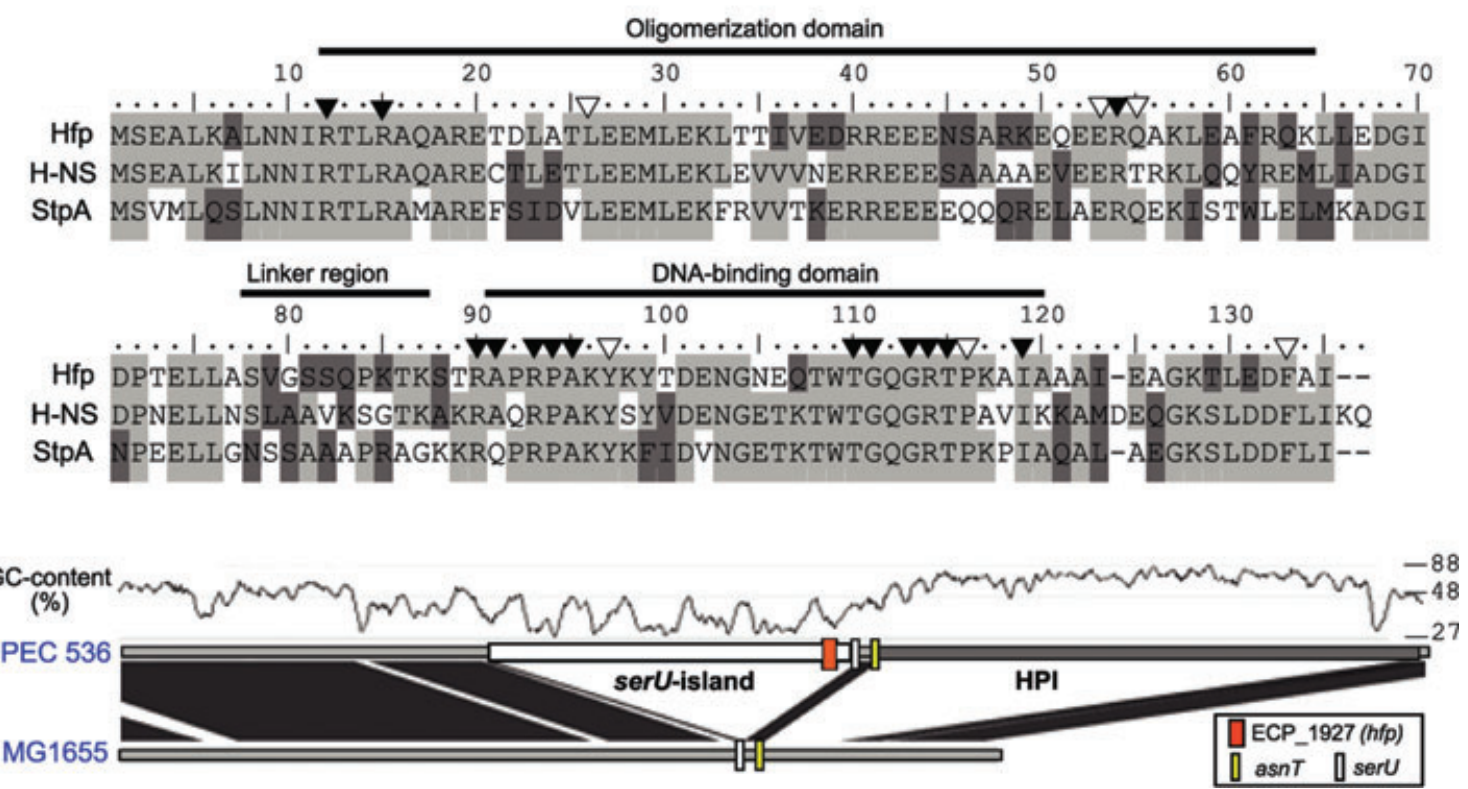

Fig. 1. Comparison of amino acid sequences and chromosomal location of the hfp gene.

A. Alignment of the three nucleoid-associated proteins of UPEC strain 536 (H-NS = ECP_1284; StpA = ECP_2632; Hfp = ECP_1927). Identical residues are depicted with light shading; dark shading indicates residues with $>50 \%$ similarity. The domain structure is indicated above the sequence. Residues that are essential for the function of H-NS are marked with filled arrowheads; residues that confer a dominant-negative phenotype when substituted are marked with open arrowheads [as summarized by Williams and Rimsky (1997)].

B. Genome comparison of the hfp region of UPEC strain 536 and non-pathogenic E. coli K-12 strain MG1655. Dark shading indicates regions of high homology, while horizontally acquired sequences can be identified as white regions with no homology. The localization of the hfp gene in the genome of the pathogen, as well as of tRNA loci is marked within the sequences. The corresponding serU-associated genomic island is located in close vicinity to the 'high pathogenicity island' (HPI) (Carniel, 2001). The GC content of the UPEC strain 536 sequence is depicted at a window size of 500 nucleotides.

\section{Hfp is directly interacting with the H-NS protein}

To test whether the $h f p$ gene encodes a functional gene product, plasmid pCM9 carrying the hfp gene and an approximately $200 \mathrm{nt}$ upstream region was introduced into hns mutants of the K-12 strains MG1655 and MC4100, and its ability to complement for the lack of $\mathrm{H}$-NS was assessed in the cases of some well-described hns mutant phenotypes (Fig. S1). In brief, we provide evidence that the $h f p$ gene produces a functional protein

Table 1. Occurrence of the hfp gene in different $E$. coli variants. ${ }^{a}$

\begin{tabular}{lrrc} 
Variant & $h f p^{+}$ & $h f p^{-}$ & $\%$ hf \\
\hline ExPEC $(n=67)$ & 39 & 28 & 58.2 \\
whereof UPEC $(n=42)$ & 33 & 9 & 78.6 \\
Faecal isolates $(n=39)$ & 15 & 24 & 38.5 \\
whereof known ECOR B2 $(n=11)$ & 5 & 6 & 45.5 \\
IPEC $(n=27)$ & 1 & 26 & 3.7 \\
Total $(n=133)$ & 55 & 78 & 41.4 \\
\hline
\end{tabular}

a. The presence of the $h f p$ gene was monitored in clinical isolates by PCR screening using primers Hfp-RT1 and Hfp-RT2. Extraintestinal pathogenic $E$. coli (ExPEC) included uropathogenic $E$. coli isolates $(n=42)$, strains causing sepsis $(n=15)$ and newborn meningitis ( $n=10)$; intestinal pathogenic $E$. coli (IPEC) included enterotoxigenic $(n=4)$, enterohaemorrhagic $(n=12)$, enteropathogenic $(n=4)$, enteroinvasive $(n=2)$ and enteroaggregative $E$. coli $(n=5)$. which can complement for the lack of H-NS in terms of several H-NS-dependent operons.

Heteromeric interactions between H-NS and its homologue StpA have been described (Johansson et al., 2001). Evidence for a direct protein-protein interaction between $\mathrm{H}-\mathrm{NS}$ and Hfp were obtained when a His-tagged version of the Hfp protein was overexpressed and purified using $\mathrm{Ni}$-affinity chromatography. When the $16 \mathrm{kDa}$ His-Hfp protein was eluted from the chromatography column, a second protein of approximately $15.5 \mathrm{kDa}$ was co-purified in equimolar ratio (Fig. 2A). N-terminal sequencing of the smaller protein resulted in the sequence SEALKILM, which matches the H-NS sequence. Immunoblotting with antiserum recognizing $\mathrm{H}-\mathrm{NS}$ confirmed its presence in all purification fractions (data not shown). When performing the same purification procedure in an hns mutant of the expression strain BL21, only the $16 \mathrm{kDa}$ protein corresponding to His-tagged Hfp appeared in the eluate (Fig. 2A), thereby confirming that H-NS was co-purified in the first case. These results strongly indicate that Hfp, like its homologue StpA, is directly interacting with H-NS.

Next, heteromeric complexes were directly detected by chemical cross-linking of proteins in the eluate. Immunoblotting revealed the presence of a $30 \mathrm{kDa}$ H-NS-Hfp dimeric complex as well as higher oligomers in the cross- 

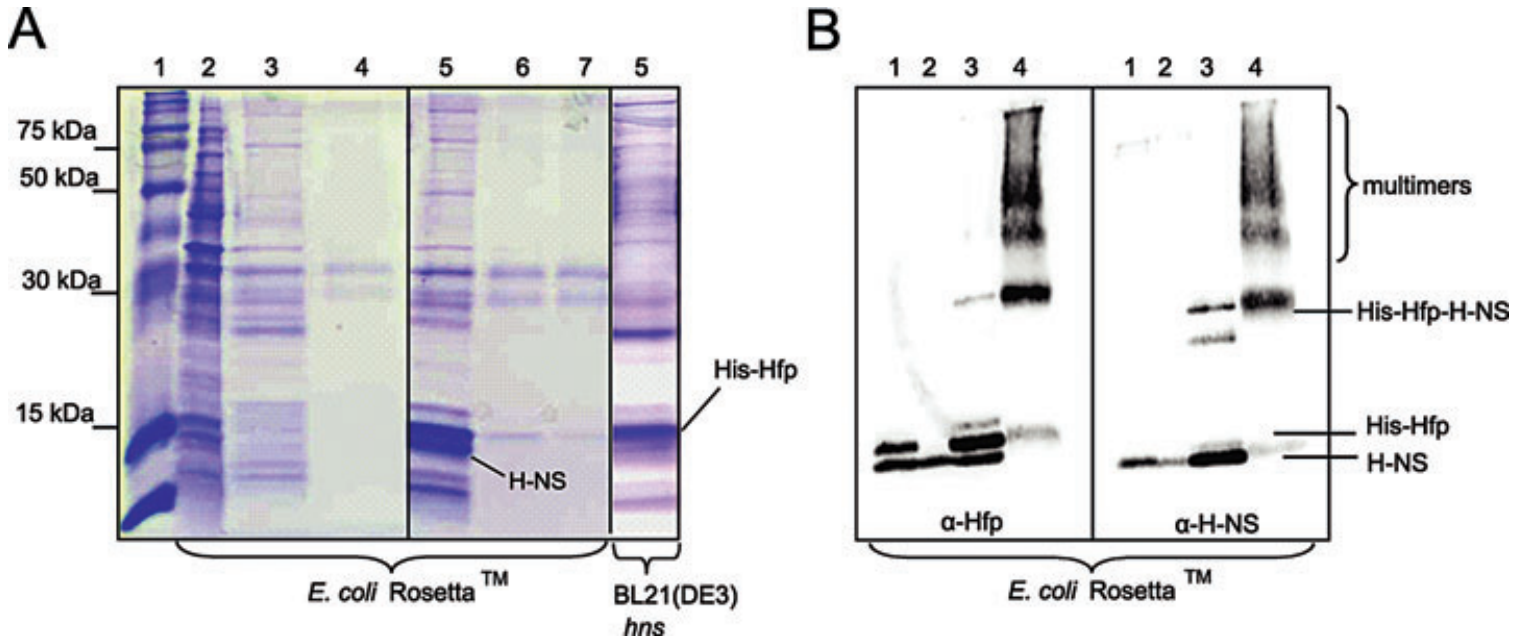

Fig. 2. Co-purification and heteromerization of H-NS and His-tagged Hfp protein.

A. Coomassie blue stain of purification fractions of His-tagged Hfp protein expressed in E. coli Rosetta ${ }^{\mathrm{TM}}$ (left and centre panel) and an $h n s$ mutant of $E$. coli $\mathrm{BL} 21$ (DE3) (right panel) respectively. The protein was overexpressed from plasmid $\mathrm{pCM} 8$ and purified using $\mathrm{Ni}^{2+}$-affinity chromatography columns. Lanes in all panels: $1=$ standard (Rainbow Marker RPN 800, GE Healthcare); $2=$ column load; $3=$ flow through; $4=$ column wash; $5=$ first fraction of eluate; $6=$ second fraction of eluate; $7=$ third fraction of eluate.

B. Immunoblot of purification fractions from E. coli Rosetta ${ }^{\mathrm{TM}}$ after chemical cross-linking with $2.5 \mathrm{mM}$ glutaraldehyde. Polyclonal antibodies reacting with $\mathrm{Hfp}$ (left panel) or $\mathrm{H}-\mathrm{NS}$ (right panel), were used for detection of oligomers in cross-linked samples. Lanes in both panels: 1 = column load; 2 = flow trough; 3 = eluate before cross-linking; 4 = eluate after cross-linking. Protein bands that matched the predicted molecular weight of individual monomers, dimers and multimers are labelled accordingly. The identity of other protein bands of lower molecular weight in the crude eluate, which were also recognized by the polyclonal antiserum, remains unclear.

linked fraction (Fig. 2B). To investigate if this heteromerization with $\mathrm{H}-\mathrm{NS}$ has a stabilizing function for $\mathrm{Hfp}$, as it has been shown for StpA (Johansson and Uhlin, 1999), protein stability was analysed by expressing the native Hfp protein from plasmid pCM9 in E. coli MC4100 derivatives lacking both StpA and H-NS, and/or the Lon protease. No degradation of $\mathrm{Hfp}$ in an $\mathrm{hns}^{-} \mathrm{lon}^{+}$strain could be observed $3 \mathrm{~h}$ after stopping protein synthesis by addition of spectinomycin (Fig. S2), which suggests that the Hfp protein, in contrast to StpA, is stable even in absence of its interaction partner H-NS.

\section{Hfp and StpA are required for normal growth at low or high temperatures}

To study the biological role of the H-NS homologues Hfp and StpA, a series of derivatives with mutations of the $h f p, h n s$ and $\operatorname{stp} A$ loci was constructed in UPEC strain 536. As a first phenotypical test, the growth ability of those strains was assessed on agar plates or in liquid medium at a temperature range between $25^{\circ} \mathrm{C}$ and $45^{\circ} \mathrm{C}$. The data are summarized in Fig. $\mathrm{S} 3$ and in Table 2. The most intriguing results were obtained with

Table 2. Phenotypic characterization of mutants.

\begin{tabular}{|c|c|c|c|c|c|c|c|c|}
\hline Phenotype & $536 \mathrm{wt}$ & $\operatorname{stp} A$ & hns & $h f p$ & $h n s / \operatorname{stp} A$ & $h f p / s t p A$ & $h n s / h f p$ & $h f p / h n s / \operatorname{stp} A$ \\
\hline bgl phenotype & - & - & + & - & + & - & + & + \\
\hline Motility & + & + & - & + & - & + & - & - \\
\hline Haemolytic activity $\left(30^{\circ} \mathrm{C}\right)$ & 14 & 15 & 85 & 14 & 76 & 18 & 127 & 70 \\
\hline Curli fimbriae: $37^{\circ} \mathrm{C}$ & - & - & + & - & + & - & + & + \\
\hline $25^{\circ} \mathrm{C}$ & + & + & - & + & - & + & - & - \\
\hline Autoaggregation & - & - & + & - & ++ & - & ++t & +H+ \\
\hline Siderophore kinetics & Slow & Slow & Fast & Slow & Fast & Slow & Fast & Fast \\
\hline Generation time $(\mathrm{min}): 25^{\circ} \mathrm{C}$ & 51 & 50 & 55 & 50 & 62 & 49 & 111 & 83 \\
\hline $37^{\circ} \mathrm{C}$ & 24 & 24 & 29 & 25 & 47 & 24 & 27 & 53 \\
\hline $45^{\circ} \mathrm{C}$ & 30 & 28 & 27 & 29 & 103 & 29 & 29 & 145 \\
\hline
\end{tabular}

Assay systems: Generation times were assessed from cultures grown in LB medium at indicated temperatures with aeration; the bg/ phenotype was assessed on agar plates containing the substrate X-Glu; motility was assessed on $0.3 \%$ agar plates; haemolytic activity was measured from culture supernatants and is defined as the dilution at which $50 \%$ haemolysis occurred; expression of curli fimbriae was assessed on agar plates containing congored dye; assays for autoaggregation and siderophore production were performed as described in Experimental procedures. 
A
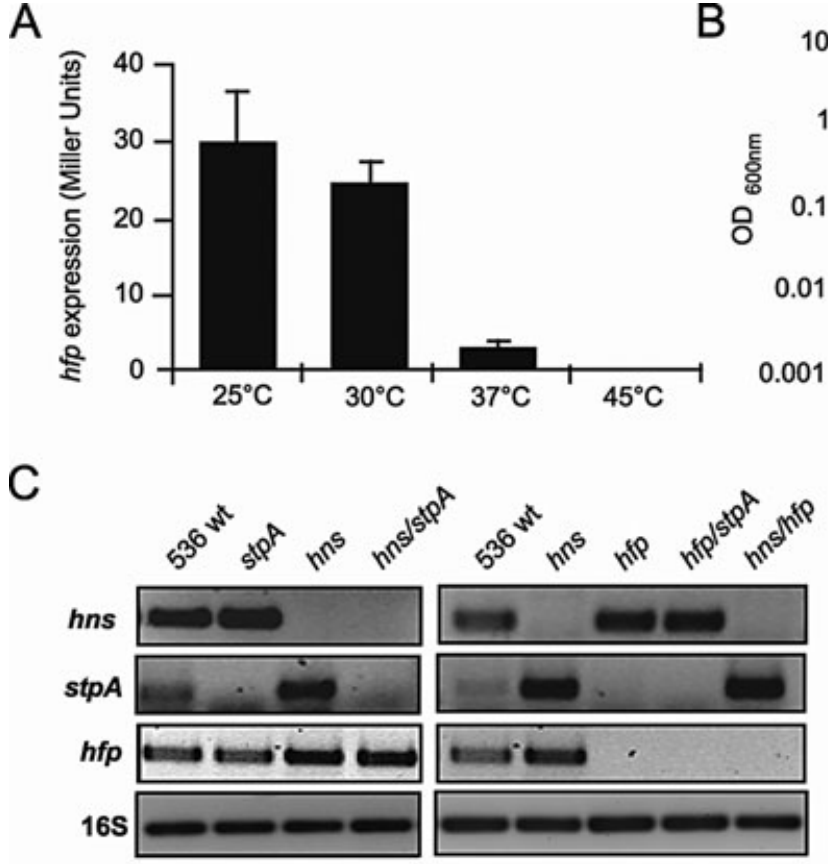

B

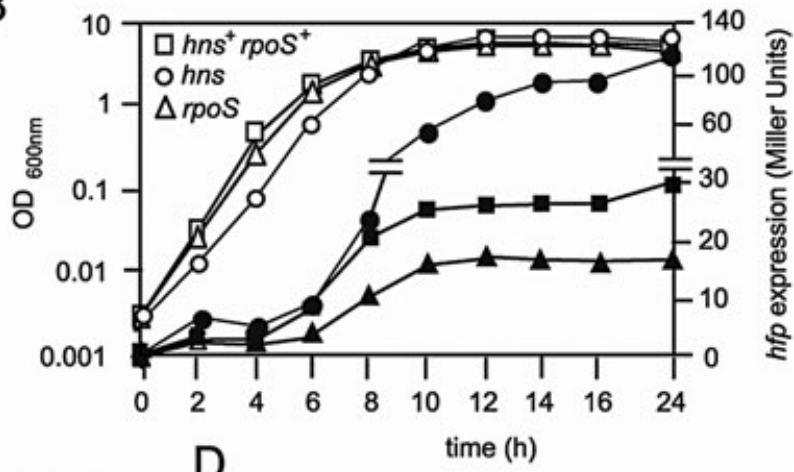

Fig. 3. Analysis of $h f p$ transcription and transcriptional cross-regulation.

A. Expression of the $h f p$ gene in response to temperature determined in a chromosomal $536 \mathrm{hfp}-\mathrm{lacZ}$ fusion strain. Bacteria were grown in LB medium at a temperature range from $25^{\circ} \mathrm{C}$ to $45^{\circ} \mathrm{C}$. Samples for $\beta$-galactosidase measurements were taken after $16 \mathrm{~h}$ of incubation. Mean values and standard deviations of three independent experiments are shown.

B. Expression of the $h f p$ gene throughout the growth curve. The chromosomal $h f p-l a c Z$ fusion strain (squares) and its otherwise isogenic $h n s$ (circles) and $r p o S$ (triangles) mutant derivatives were grown at $30^{\circ} \mathrm{C}$ in LB medium. Every second hour, the optical density of the culture (open symbols) and $\beta$-galactosidase activity (closed symbols) of the cultures were measured and plotted in the same graph.

C. Transcript amounts of $h n s, \operatorname{stp} A$ and $h f p$ in strain 536 and isogenic mutants lacking one or two of the nucleoid-associated proteins were analysed by semi-quantitative RT-PCR using primer pairs binding in the coding sequences of $h n s$, stpA and $h f p$. RNA samples were prepared from mid-log-phase cultures grown at $37^{\circ} \mathrm{C}$. 16S rRNA was used for normalization of CDNA amounts. PCR reactions were performed in triplicate on two biological replicates per sample. The images illustrate sections of ethidium bromide-stained gels from representative experiments with each primer combination.

D. Expression of the $h f p$ gene as determined by measuring $\beta$-galactosidase activity from a chromosomal $h f p-l a c Z$ fusion. The parental strain (wt), as well as isogenic $h n s$ and $\operatorname{stp} A$ mutants grown at $30^{\circ} \mathrm{C}$ for $16 \mathrm{~h}$, was analysed. Mean values and standard deviations of three independent experiments are shown.

the $h n s / s t p A$ and the $h n s / h f p$ double mutants at the lowest and highest temperatures: in liquid medium at $45^{\circ} \mathrm{C}$, the hns $/$ stpA mutant exhibited a severe growth defect, with a 3.5 times longer generation time than that of the hns/hfp mutant (103 min versus $29 \mathrm{~min}$ respectively). The opposite was true at $25^{\circ} \mathrm{C}$, where the hns/ hfp mutant grew very poorly, while at this temperature, the generation time of the hns/stpA mutant was 1.8 times shorter in comparison with the hns/hfp mutant (62 min versus $111 \mathrm{~min}$ respectively). Taken together, our results show that $h n s / \operatorname{stp} A$ and $h n s / h f p$ double mutants differ drastically in their growth ability at the $45^{\circ} \mathrm{C}$ and $25^{\circ} \mathrm{C}$ growth temperatures, which represents the first H-NS-independent phenotype of these mutants. This suggests that - in the absence of $\mathrm{H}-\mathrm{NS}$ - functional StpA protein is required for growth at temperatures above body temperature, while Hfp promotes growth at room temperature instead.
The stpA and hfp genes are diametrically expressed with changing environmental conditions

The results derived from the growth experiments suggested distinct requirements for Hfp and StpA at low or high temperatures respectively. Therefore, it was investigated whether these differences in the requirement are reflected in the expression levels of the corresponding genes at those temperatures. When studying hfp expression using a chromosomal $h f p-l a c Z$ fusion derivative of UPEC strain 536 at a temperature range from $25^{\circ} \mathrm{C}$ to $45^{\circ} \mathrm{C}$, a significant reduction in $h f p$ expression could be observed with increasing temperature (Fig. 3A). While the hfp gene was clearly expressed at both $25^{\circ} \mathrm{C}$ and $30^{\circ} \mathrm{C}, \beta$-galactosidase activity dropped markedly when cultures were grown at $37^{\circ} \mathrm{C}$ and was undetectable in cultures grown at $45^{\circ} \mathrm{C}$.

The transcript levels of $h n s, \operatorname{stp} A$ and $h f p$ were also determined by reverse transcription-PCR (RT-PCR) using 
RNA samples from wild-type (wt) strain 536 grown at a temperature range of $25-45^{\circ} \mathrm{C}$. Relatively constant levels of hns transcript were observed at every temperature, while stpA transcript levels increased with increasing temperature (data not shown), thereby indicating that the temperature-regulated expression of $\operatorname{stp} A$ described in E. coli K-12 (Free and Dorman, 1997) also occurs in pathogenic isolates. On the other hand, the level of $h f p$ transcript decreased with increasing temperature in agreement with the $h f p-l a c Z$ fusion data. Moreover, we observed a clear difference in the abundance of the transcripts in the wt at $37^{\circ} \mathrm{C}$ when compared with each other, with $h n s$ being much more abundant than stpA or hfp (see also Fig. $3 \mathrm{C}$, first lane). This is consistent with previous observations that $\operatorname{stp} A$ levels are usually low at $37^{\circ} \mathrm{C}$ (Sondén and Uhlin, 1996; Zhang et al., 1996) and indicates that the same holds true for $h f p$.

The expression of $\operatorname{stp} A$ is growth phase dependent and occurs transiently during exponential growth (Free and Dorman, 1997). To analyse expression of the hfp gene through the batch growth phases, $\beta$-galactosidase activity from the chromosomally encoded $h f p-l a c Z$ fusion was measured every second hour during growth at $30^{\circ} \mathrm{C}$. Only basal $h f p$ expression levels could be detected throughout logarithmic phase (Fig. 3B). However, $\beta$-galactosidase activity markedly increased upon entry into stationary phase and stayed constant thereafter. The observed stationary-phase induction of $h f p$ does not seem to be strictly dependent on RpoS, an alternative sigma-factor regulating many genes expressed in stationary phase. Expression of $h f p$ in an rpos mutant derivative of the $h f p-l a c Z$ fusion strain was significantly reduced $(60 \%$ reduction compared with $\mathrm{wt}$ ) but followed the same pattern of induction upon entry into stationary phase as observed in the wt (Fig. 3B). Temperature and growth phase are not the only factors that divergently regulate the expression of the H-NS homologues. Table S1 in Supporting information lists the differences between the expression of the stpA gene and the hfp gene as described in the literature and according to our present studies. In summary, we provide evidence that the expression levels of the H-NS-related genes hfp and $\operatorname{stpA}$, which are relatively low during standard growth at $37^{\circ} \mathrm{C}$, can be induced at certain, seemingly diametrical conditions, e.g. by temperature variations. Moreover, the results suggest that Hfp and StpA probably operate at temperatures other than $37^{\circ} \mathrm{C}$.

\section{Expression of the hfp, stpA and hns genes is subject to negative auto- and cross-regulation}

Next, the interplay between the nucleoid-associated proteins at the transcriptional level was investigated. H-NS and StpA have been shown to negatively regulate tran- scription of their own genes (autoregulation) as well as of their homologue (cross-regulation). To include Hfp, transcript amounts of all three nucleoid-associated proteinsencoding genes were detected by semi-quantitative RT-PCR in UPEC strain 536 and the hfp, hns and stpA mutant derivatives (Fig. 3C). De-repression of both $h f p$ and $\operatorname{stp} A$ transcription occurred in the absence of $\mathrm{H}-\mathrm{NS}$, thereby indicating a role for $\mathrm{H}-\mathrm{NS}$ as a repressor of both its homologues, in agreement with published data (Sondén and Uhlin, 1996). The negative effect of H-NS on hfp expression was also observed using an hns mutant derivative of the $h f p-l a c Z$ fusion strain (Fig. 3B and D). A slightly higher amount of hns transcript could be detected in the stpA mutant, as it was described before in E. coli $\mathrm{K}-12$ (Sondén and Uhlin, 1996), but also in mutants lacking Hfp, suggesting that both StpA and Hfp negatively affect $h n s$ expression. A lack of StpA did not affect hfp transcription; vice versa, expression of $\operatorname{stp} A$ was not altered in an $h f p$ mutant. To further quantify the effect of StpA on hfp expression, a mutation in the stpA gene was introduced into the $h f p-l a c Z$ fusion strain and $\beta$-galactosidase activity was measured from cultures grown at $30^{\circ} \mathrm{C}$ for $16 \mathrm{~h}$ (Fig. 3D). In accordance with the RT-PCR results (Fig. 3C), no alteration in $h f p$ expression was observed in the stpA mutant background, which corroborates disparate effects of $\mathrm{H}-\mathrm{NS}$ and StpA on hfp expression.

\section{The hfp promoter region is target for DNA binding by nucleoid-associated proteins}

The repressive effect of $\mathrm{H}-\mathrm{NS}$-like proteins on target gene expression involves DNA binding to promoter regions, which also might be the mechanism for the transcriptional cross-regulation described above. To first characterize the $h f p$ promoter region, various parts of the upstream region of the hfp gene were fused to a plasmid-encoded lacZ gene and transcriptional activity at different growth stages was assessed in a lac Z mutant derivative of strain 536 (Fig. S4A). In brief, the results indicated that transcriptional activity required upstream sequences of more than 70 nucleotides in length, with maximal activity measured with a construct encompassing 412 nucleotides upstream of the translational start codon, and confirmed both the growth phase-dependent and H-NS-regulated expression of $h f p$.

To further characterize the hfp promoter region, the start point of $h f p$ transcription was determined by primer extension analysis. The most prominent transcriptional start point was identified at a $T$ residue $339 \mathrm{bp}$ upstream of the hfp translation initiation codon (see Fig. S5). Transcription from this promoter presumably is repressed by $\mathrm{H}-\mathrm{NS}$, since the amount of extension product was significantly increased in samples derived from an hns mutant. Analysis of the preceding sequence revealed a region of high 

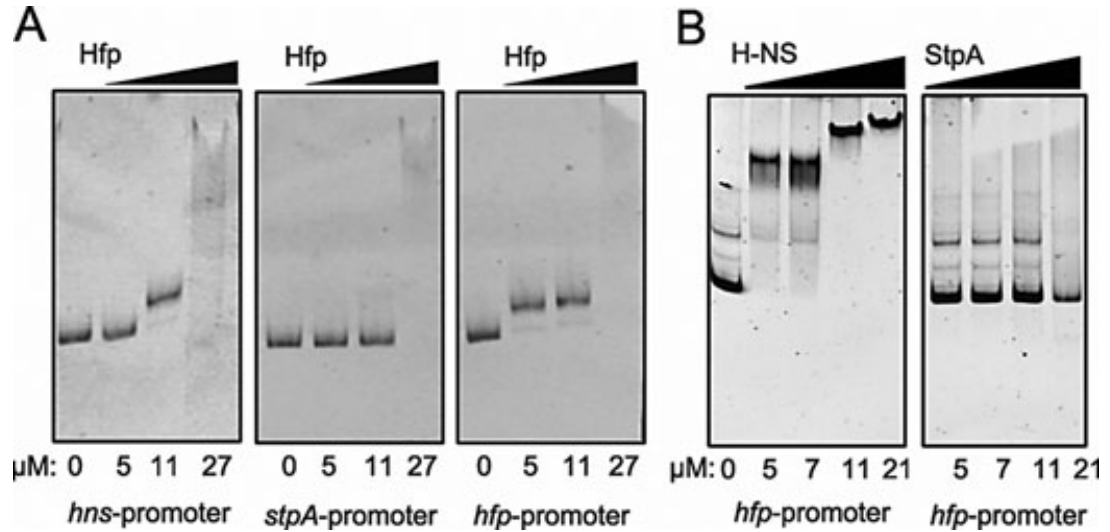

Fig. 4. DNA-binding pattern of Hfp, H-NS and StpA to cognate promoter regions determined by electrophoretic mobility shift analysis. A. Binding activity of purified Hfp protein to PCR-generated DNA fragments spanning the promoter regions of $h n s, \operatorname{stp} A$ and $h f p$. The figure represents ethidium bromide-stained acrylamide gels after digital inversion of the images. Protein was added at the concentrations indicated below each lane in a range from 0 to $27 \mu \mathrm{M}$.

B. Comparison of DNA-binding affinities of $\mathrm{H}-\mathrm{NS}$ and StpA at the hfp promoter region. The assay was carried out as described before, using the same DNA fragment as in (A). The purified proteins were added in a concentration range of $0-21 \mu \mathrm{M}$. Images depict representative gels of at least two independent experiments.

similarity to the proposed H-NS binding motif (Lang et al., 2007) between the -10 and -35 elements, which would explain the observed H-NS-mediated repression of $h f p$ expression. A second transcriptional start site with an RpoS-dependent -10 box was predicted 87 bp upstream of the translation initiation codon, but the transcript was barely detectable by primer extension analyses. This proximal promoter also contained a predicted Fis site (Fig. S5), which might explain the modest degree of activation of $h f p$ expression observed using $h f p-l a c Z$ reporter fusion derivatives (Table $\mathrm{S} 1$ and data not shown). Taken together, these results suggest that $h f p$ is transcribed monocistronically from promoters located within a region of -70 to -412 nucleotides counting backwards from ATG and that several global regulators might be involved in regulating $h f p$ expression.

$\mathrm{H}$-NS was shown to preferentially bind to AT-rich, intrinsically curved DNA sequences (Yamada et al., 1990; Owen-Hughes et al., 1992). The AT content of a 400-bplong upstream region of $h f p$ was calculated to be $63.8 \%$, which is similar to the AT content of the corresponding hns and $\operatorname{stp} A$ upstream regions $(60.6 \%$ and $63.5 \%$ AT respectively). A computer prediction software suggested strong intrinsic curvature of the 400 bp fragment upstream of hfp (data not shown). This apparent feature, and the presence of the predicted $\mathrm{H}$-NS binding site, made us hypothesize that the $h f p$ promoter region is a potential target for binding of nucleoid-associated proteins. To elucidate the DNA-binding activities of all three nucleoidassociated proteins to the promoter regions of $h f p, h n s$ and $\operatorname{stp} A$, electrophoretic mobility shift assays (EMSA) were performed. Purified Hfp protein caused a minor shift of DNA fragments containing the $h f p$ promoter or the $h n s$ promoter region, respectively, at a protein concentration of 5-11 $\mu \mathrm{M}$, while no such shift was observed with the stpA promoter fragment (Fig. 4A). When studying the potential for H-NS- or StpA-mediated cross-regulation at the $h f p$ promoter, H-NS was found to bind to the $h f p$ fragment in a cooperative manner, resulting in extensive, stepwise shifts (Fig. 4B). No specific binding could be observed with purified StpA protein at the same conditions, thereby supporting the result of unaltered $h f p$ expression in the stpA mutant (Fig. 3D). The DNA-binding activity of the StpA protein preparation was verified in a control experiment where binding of StpA was monitored (data not shown) to a DNA fragment representing the promoter of the E. coli K-12 stpA locus (Sondén and Uhlin, 1996). In summary, our results with DNA representing the $h f p$ and $h n s$ promoter regions suggested that $\mathrm{Hfp}$ has a recognizable albeit weak DNA-binding activity. Furthermore, the difference in the shifts caused by H-NS and $\mathrm{Hfp}$ at a given protein concentration indicated that there would be differences in the arrangement or the stability of the DNA-protein complexes formed by the two proteins.

\section{Differential modulation of gene expression by $\mathrm{Hfp}$ and $\operatorname{StpA}$}

The data obtained from transcriptional analysis suggested that the effects of $\mathrm{Hfp}$ and $\operatorname{StpA}$ are the strongest at temperatures other than $37^{\circ} \mathrm{C}$. This was also confirmed by the observation that both the $h f p$ and the $\operatorname{stp} A$ single mutants exhibited no obvious phenotype when tested for the expression of the known major virulence traits of strain 536 at $37^{\circ} \mathrm{C}$ (Table 2). Lack of H-NS on the other hand affected the expression of all factors tested, resulting in a hyper-fimbriated, hyper-haemolytic, but non-motile phenotype at $37^{\circ} \mathrm{C}$, as described before (Müller et al., 2006). 
The phenotypes at $37^{\circ} \mathrm{C}$ of the double and triple mutants were solely dependent upon $\mathrm{H}-\mathrm{NS}$ but not of its homologues StpA or Hfp at that temperature.

Fimbrial adhesins are crucial factors for virulence of uropathogenic E. coli. When comparing expression of two of the fimbrial operons of UPEC strain 536 in the hns/stpA and the hns/hfp mutant, a clear difference was observed: as determined from the immunoblot analyses of samples from $37^{\circ} \mathrm{C}$, lack of StpA resulted in a less pronounced de-repression of Prf-pili in the absence of $\mathrm{H}-\mathrm{NS}$, whereas expression of Sfa-pili was even higher compared with the hns single and hns/hfp double mutants (Fig. 5A; middle panel), which indicates differences in the ability of StpA and $\mathrm{Hfp}$ to modulate fimbriation at $37^{\circ} \mathrm{C}$. The expression of fimbriae was also tested at $25^{\circ} \mathrm{C}$ and $45^{\circ} \mathrm{C}$ (Fig. $5 \mathrm{~A}$; left and right panel respectively). A de-repression of the Sfapili in strains lacking $\mathrm{H}-\mathrm{NS}$ could be observed at $25^{\circ} \mathrm{C}$, as described for $37^{\circ} \mathrm{C}$ (Fig. $5 \mathrm{~A}$; middle panel), while at $45^{\circ} \mathrm{C}$ Sfa-pili were only produced by the $h n s / h f p$ double mutant, which indicates that StpA alone was not able to repress sfa expression at this temperature. Prf-pili could not be detected in any of the strains at $25^{\circ} \mathrm{C}$ and $45^{\circ} \mathrm{C}$. This seems consistent with a tight regulation of the expression of those virulence traits that would be optimized to the conditions in a mammalian host, and also suggests that StpA and Hfp probably do not play any major role in regulation of virulence factor expression in that specific environmental setting.

The expression of the capsule determinant was investigated as a second potential target operon, since H-NS was shown to affect the expression of the region 1 genes of the $\mathrm{K} 5$ capsule in a temperature-dependent manner by repressing transcription at $25^{\circ} \mathrm{C}$, but being required for maximum expression at $37^{\circ} \mathrm{C}$ (Corbett et al., 2007). The role of the nucleoid-associated proteins in the production of capsular polysaccharide in UPEC strain 536 was assessed by ELISA. In 536 wt bacteria, the highest expression level of capsular polysaccharide was detected at $37^{\circ} \mathrm{C}$, in agreement with published data (Corbett et al., 2007), whereas the expression level dropped to $60 \%$ at $44^{\circ} \mathrm{C}$, and only background levels were reached at $25^{\circ} \mathrm{C}$ (Fig. 5B). When the mutant series was analysed, only the hns and hns/hfp double mutants (but not the hns/stpA mutant) showed alterations in capsule expression. At $25^{\circ} \mathrm{C}$, reactivity with the $\mathrm{K} 15$ antiserum was 3.1 -fold and 4.5-fold increased in the hns and hns/hfp mutants, respectively, as compared with wt, which also coincided with a mucoid phenotype of these mutants at this temperature on plates. At $37^{\circ} \mathrm{C}$, all mutants behaved like the wt, except the hns/hfp mutant that showed a slightly but significantly higher capsule production. At $44^{\circ} \mathrm{C}$, only the hns mutant yielded significantly higher values as compared with wt. These results confirm a role of nucleoidassociated proteins in the production of the capsular polysaccharide of serotype $\mathrm{K} 15$ in a temperaturedependent manner.

To further characterize the roles of Hfp and StpA in transcription, a reporter system for bgl expression was used as a well-described target for regulation by $\mathrm{H}-\mathrm{NS}$-like proteins. A plasmid containing a transcriptional bg/G::lacZ fusion (pKENV61) was introduced into strain 536 and the set of mutants and $\beta$-galactosidase activity was measured from cultures grown at $25^{\circ} \mathrm{C}, 37^{\circ} \mathrm{C}$ or $42^{\circ} \mathrm{C}$ (Fig. $5 \mathrm{C}$ and D). Since the strains used are all $\mathrm{lac}^{+}$on the chromosome, a control experiment was performed using plasmid pKENV61 1 , where a part of the bglG::lacZ fusion was deleted. In all strains and conditions tested, the background $\beta$-galactosidase activity measured was less than 20 Miller units (data not shown). When measuring $\beta$-galactosidase activity in strains carrying pKENV61, all strains lacking $\mathrm{H}-\mathrm{NS}$ exhibited de-repressed bg/ transcription at any of the temperatures tested, with the strongest effect observed at $25^{\circ} \mathrm{C}$ (Fig. $5 \mathrm{C}$ and D). Interestingly, bgl expression was four to five times higher in the hns/stpA double mutant compared with the hns single mutant at $37^{\circ} \mathrm{C}$ and $42^{\circ} \mathrm{C}$, but not at $25^{\circ} \mathrm{C}$ (Fig. 5D). The same effect was observed in an $h n s^{+}$background, where de-repression in both $\operatorname{stp} A$ and hfp/stpA mutants compared with the wt increased with increasing temperatures (Fig. 5C). These results suggest that StpA has an additive effect in the repression of the $b g /$ operon and that the strength of repression correlates with the expression levels of the $\operatorname{stp} A$ gene, i.e. high StpA levels at $42^{\circ} \mathrm{C}$ coincide with a strong repressive effect on $b g /$ transcription. Lack of $\mathrm{Hfp}$ alone did not result in de-repression of the $\mathrm{bgl}$ operon (Fig. 5C). However, lack of both H-NS and Hfp resulted in higher $\beta$-galactosidase values than those obtained by the lack of $\mathrm{H}-\mathrm{NS}$ alone, and this additional increase in $\mathrm{bgl}$ expression was the highest at $25^{\circ} \mathrm{C}$, where the difference between the $h n s / h f p$ and the $h n s$ single mutant was more than 1000 Miller units (Fig. 5D). From this, we can conclude that $\mathrm{Hfp}$, in contrast to StpA, does not contribute to repression of the $b g /$ operon when $\mathrm{H}$-NS is present. In the absence of H-NS, however, Hfp can exert a repressive effect that is the strongest at low temperatures, two conditions where Hfp levels are induced.

Taken together, as exemplified by the bgloperon and the capsule determinant, a clear correlation could be made between $h f p$ and $\operatorname{stp} A$ transcript levels and the strength of modulatory effects of the encoded proteins on the expression of $\mathrm{H}-\mathrm{NS}$-regulated genes. This suggests that the $\mathrm{H}-\mathrm{NS}$ homologues Hfp and StpA can act as modulators of gene expression at divergent environmental conditions.

\section{Discussion}

In this study, we provide evidence that many ExPEC, as exemplified by UPEC strain 536, encode three members of 
A

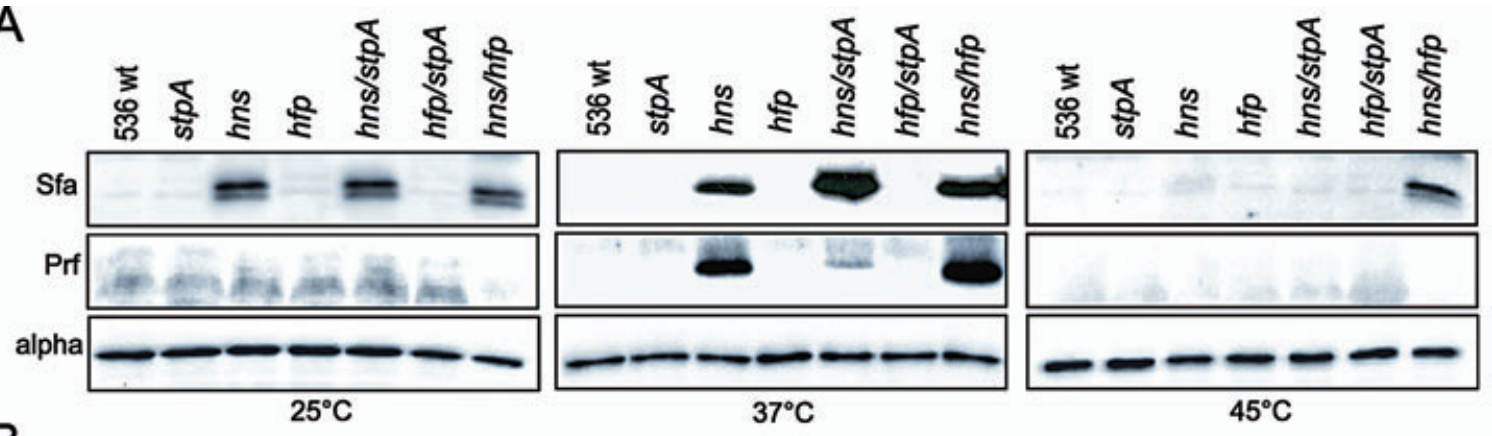

B

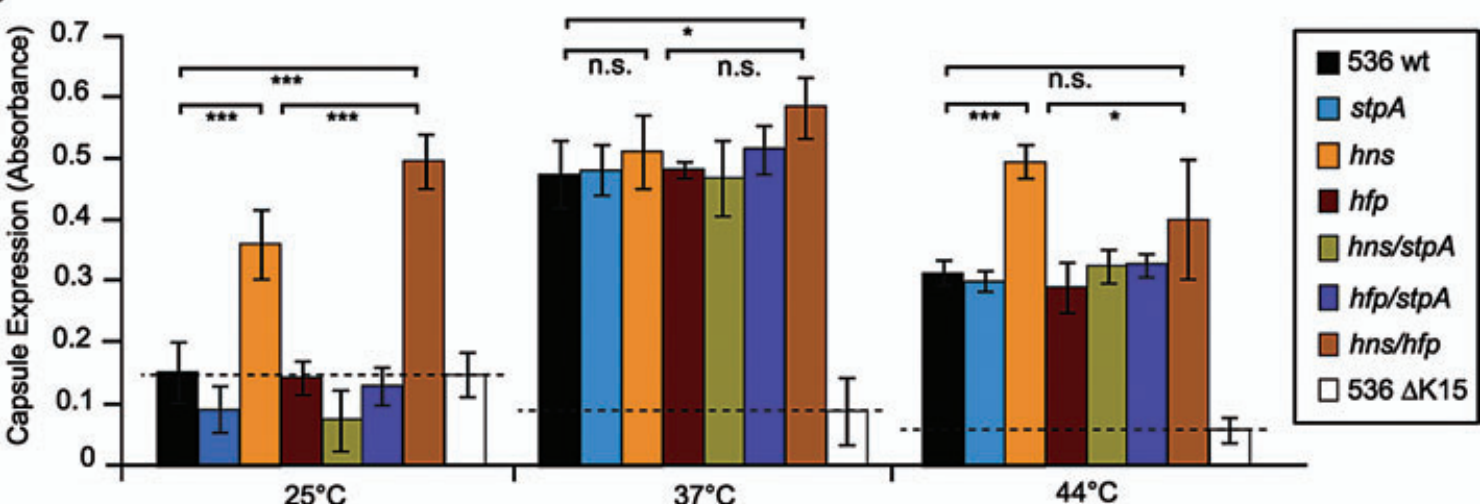

C

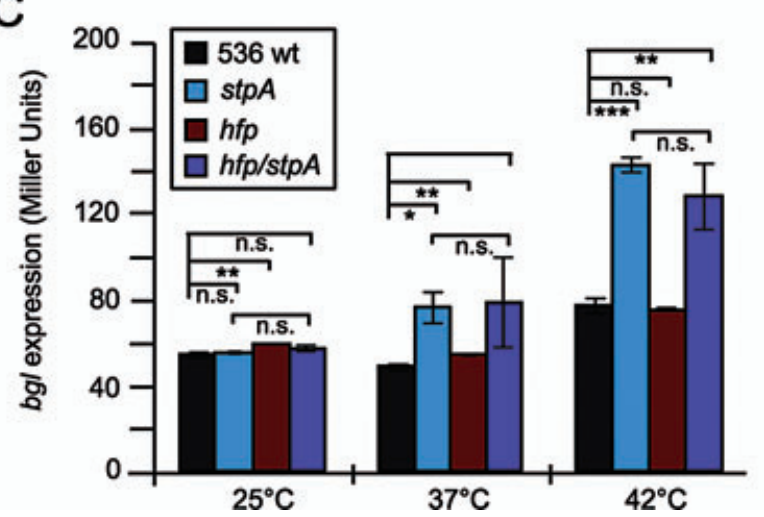

D

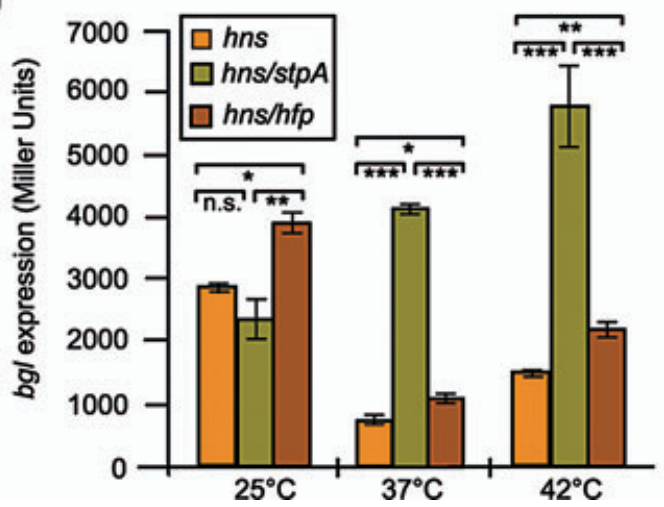

Fig. 5. Effect of Hfp and StpA on gene expression.

A. Effect of the lack of nucleoid-associated proteins on fimbriation. The protein levels of the major subunits of S and P fimbriae (SfaA and PrfA respectively) were determined by Western blotting from cultures of UPEC strain 536 and mutants lacking one or two of the

nucleoid-associated proteins $\mathrm{H}$-NS, Hfp and StpA. All strains were grown to mid-log phase at $25^{\circ} \mathrm{C}, 37^{\circ} \mathrm{C}$ and $45^{\circ} \mathrm{C}$ respectively. The alpha-subunit of the RNA polymerase was used as a control for equal loading of whole cell lysates. Images depict representative blots of at least two independent experiments.

B. Effect of the H-NS homologues on the production of capsular polysaccharide on the cell surface. Strain 536 and the series of isogenic hns, $\operatorname{stp} A$ and $h f p$ mutant derivatives were grown overnight at $25^{\circ} \mathrm{C}, 37^{\circ} \mathrm{C}$ and $44^{\circ} \mathrm{C}$, and cultures were adjusted to the same optical density. The K15 capsule was detected by ELISA using pre-absorbed K15 capsule-specific antiserum and HRP-conjugated secondary antibodies and quantified by measuring the optical density at $490 \mathrm{~nm}$. Mean values and standard deviations from three independent experiments are shown. $\mathrm{C}$ and D. Effect of the H-NS homologues on expression from the H-NS-regulated bg/ promoter. Transcriptional activity was measured in strain 536 and isogenic mutants lacking $\operatorname{stp} A$ and/or $h f p(C)$ and the series of $h n s$ mutant derivatives (D). In both (C) and (D), all strains carried a transcriptional $b g l-l a c Z$ fusion on a plasmid (pKEN61). The strains were grown to mid-log phase at $25^{\circ} \mathrm{C}, 37^{\circ} \mathrm{C}$ and $42^{\circ} \mathrm{C}$, as indicated in the figure. Mean values and standard deviations from three independent experiments are shown.

In (B-D): Values marked with one asterisk are statistically significant with a $P$-value of $<0.05$, two asterisks indicate a $P$-value $<0.01$, and three asterisks indicate a $P$-value $<0.001$ as calculated using the Student's $t$-test.

the H-NS-like protein family, the global regulator H-NS itself, as well as two intraspecies homologues StpA and Hfp, similar to the situation described for the StpA and Sfh proteins of Shigella (Beloin et al., 2003). We suggest that the so far uncharacterized $h f p$ gene and its gene product are not to be considered simply the redundant result of gene duplication, but that Hfp represents a distinct functional member in a homeostatically regulated pool of $\mathrm{H}-\mathrm{NS}$ - 


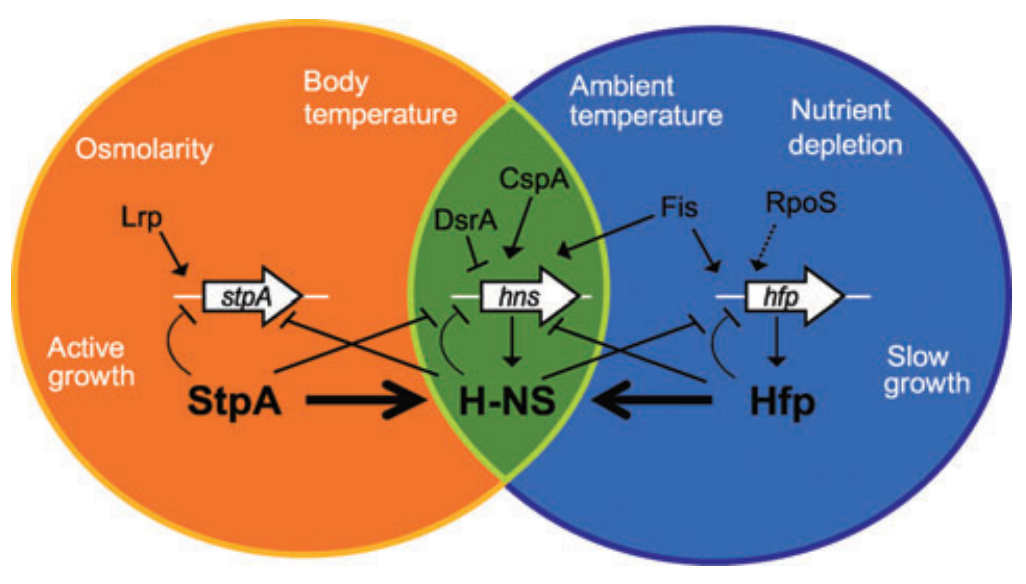

Fig. 6. Model for occupation of disparate niches by the H-NS homologues StpA and Hfp. In contrast to the abundant H-NS protein that is produced in various types of environment, expression of the stpA and hfp genes is induced by disparate environmental conditions mediated through differences in transcriptional regulation. Interactions between the H-NS-like proteins include modulating effects of both StpA and Hfp on $\mathrm{H}-\mathrm{NS}$ function by heteromerization and effects on hns expression through a complex network of transcriptional cross-regulation. like proteins. In this respect, our findings support existing hypotheses (Dorman, 2004). This protein pool includes full-length H-NS homologues such as StpA or Hfp, and, as shown by Juarez and co-workers (Madrid et al., 2007), also more distant homologues like members of the Hha/ YmoA family. In particular, our study demonstrates that the composition of the pool of nucleoid-associated proteins in E. coli 536 is distinctly regulated and changes according to environmental conditions, resulting in variable levels of $\mathrm{Hfp}$ or StpA as interaction partners for the abundant H-NS protein. The latter suggestion is derived from our transcription studies of the hfp and stpA genes. The expression levels of both genes are relatively low under standard laboratory growth conditions as described here and elsewhere (Sondén and Uhlin, 1996), which might account for the apparent lack of phenotype of $h f p$, stpA or $h f p / s t p A$ double mutants at $37^{\circ} \mathrm{C}$. Presumably, it indicates that the major effects of Hfp or StpA are not exerted at this temperature. On the other hand, as summarized in Table S1, expression of the two H-NS homologues can be markedly increased under certain conditions, e.g. at lower temperature and upon entry into the stationary growth phase for $h f p$ expression, or at higher temperatures $\left(>40^{\circ} \mathrm{C}\right)$ during active growth for stpA expression. The inducing conditions for elevated Hfp and StpA levels thereby seem to be quite diametrical, suggesting that $\mathrm{Hfp}$ and StpA rarely encounter each other in the cell. Therefore, we propose a scenario in which Hfp and StpA have evolved to being divergently regulated through selective forces exerted by different environmental niches (see Fig. 6).

Despite the differences in expression levels, the biochemical parameters of the histone-like proteins are very similar. Like StpA, the Hfp protein exhibits high homology to H-NS, thereby also suggesting a similar two-domain structure. Homology between Hfp and H-NS is the highest in the region corresponding to the $\mathrm{N}$-terminal oligomerization domain, which might account for the observed heteromerization between Hfp and H-NS. In contrast to StpA, which has been reported to be readily degraded by the
Lon protease if not protected by complex formation with H-NS (Johansson and Uhlin, 1999), heteromerization with $\mathrm{H}-\mathrm{NS}$ did not seem to be required for stability of Hfp, which demonstrates distinct properties of the Hfp and StpA proteins. Heteromerization with $\mathrm{H}-\mathrm{NS}$ might be an important aspect in the role of Hfp and StpA in the cell. Due to the observed differences between $\mathrm{H}-\mathrm{NS}$ and $\mathrm{Hfp}$ in their interaction with DNA (Fig. 4), we need to consider the possibility that a heteromeric complex might exhibit yet another distinct DNA binding property. Two examples of promoters that are regulated by alternating nucleoprotein complexes is the region 1 promoter of the $\mathrm{K} 5$ capsule determinant and the promoter of the H-NS-silenced gene for the cryptic haemolysin HlyE, also known as ClyA (Westermark et al., 2000; Corbett et al., 2007; Lithgow et al., 2007). In these cases, both H-NS and the SlyA protein bind to overlapping sites within the promoter region, resulting in higher-order protein : DNA complexes, depending on the relative concentrations of the DNA binding proteins. Our results using strain 536 suggest that $\mathrm{H}-\mathrm{NS}$ represses the production of the $\mathrm{K} 15$ capsule at low temperatures, as it was described for other group 2 capsule determinants (Corbett et al., 2007). In contrast to the $\mathrm{K} 5$ capsule, $\mathrm{H}-\mathrm{NS}$ seems not to be required for maximal expression of the $\mathrm{K} 15$ capsule at $37^{\circ} \mathrm{C}$, since no reduction was observed in the $h n s$ mutant compared with the wt. At lower temperatures, on the other hand, Hfp has a clear repressive effect on the K15 determinant, since the level of de-repression in the hns/hfp mutant differed significantly from that of the hns mutant at $25^{\circ} \mathrm{C}$ (Fig. 5B). As determined by EMSA, both purified $\mathrm{H}-\mathrm{NS}$ and $\mathrm{Hfp}$ proteins were able to bind to a DNA fragment spanning the region 1 promoter (our unpublished data). Therefore, a participation of $\mathrm{Hfp}$ in the nucleoprotein complex formed at the capsule determinant might occur.

Differential effects of Hfp and StpA were observed for other operons as well, such as the fimbrial and bgl determinants. Nevertheless, most of the effects of Hfp and StpA on gene expression could be revealed when $\mathrm{H}-\mathrm{NS}$ was absent, i.e. in the $h n s / h f p$ and $h n s / \operatorname{stp} A$ double 
mutants. This might partly result from the low expression levels of $\operatorname{stp} A$ and $h f p$ in the wt due to repression by H-NS, thus rendering $\mathrm{H}-\mathrm{NS}$ much more abundant, which might mask the effects of the H-NS homologues. In the absence of $\mathrm{H}-\mathrm{NS}$, on the other hand, Hfp and StpA levels increase and they can assume some of the H-NS functions. However, the results obtained from our growth studies clearly indicate that Hfp and StpA are not simple 'molecular backups' for H-NS.

Characterization of the $\mathrm{H}-\mathrm{NS}$-like genes and proteins also involves questions about what selective forces may promote establishment of genes moved by lateral gene transfer. In this study, we also investigated the presence of the hfp gene and the serU-associated genomic island by PCR screening of a large set of different $E$. coli pathotypes and variants. The hfp gene was mainly detected in extraintestinal pathogenic isolates, but is also present in some faecal isolates. Since the faecal flora is considered to be the reservoir for uropathogenic $E$. coli, and many of the faecal isolates that carried the hfp gene belonged to ECOR group B2, the phylogenetic lineage that comprises mostly extraintestinal pathogenic isolates, the distribution pattern of $h f p$ among faecal ECOR B2 strains observed can be explained. It is also very striking that only one out of 27 IPEC strains included in our PCR screen, EAEC isolate $\mathrm{O} 42$, was positive for the hfp gene. However, both uropathogenic and faecal isolates were over-represented in the strain collection compared with IPEC strains, which could result in a certain bias. Therefore, it remains to be elucidated whether there is a specific selection for the $h f p$ gene in non-IPEC variants. Moreover, amplification of a PCR product of the right size does not imply an unaltered nucleotide sequence or a functional protein. Hence, not all hfp-positive strains can be necessarily considered to express the hfp gene.

In conclusion, many pathogenic $E$. coli isolates encode two distinct $\mathrm{H}-\mathrm{NS}$ homologues, Hfp and StpA, which differ in their function and in the expression levels of the respective genes. All three proteins exhibit distinct interactions at the transcriptional and at the protein level. These interactions result in fine-tuned expression of a broad subset of target genes, including virulence-associated genes such as fimbriae genes and the capsular polysaccharide genes in UPEC strain 536. Moreover, Hfp exemplifies a regulatory protein encoded on a genetic element acquired by lateral gene exchange which made an impact on the regulatory circuits of the cell as an integrated partner in the pool of nucleoid-associated proteins.

\section{Experimental procedures}

\section{Bacterial strains and culture conditions}

All strains and plasmids used in this study are listed in Table S2. The strain collection used for the PCR screening has been described elsewhere (Dobrindt et al., 2001). Unless stated otherwise, bacteria were grown with aeration in Luria broth (LB) at $37^{\circ} \mathrm{C}$. The temperature $44^{\circ} \mathrm{C}$ or $45^{\circ} \mathrm{C}$ was used as high temperature condition, except when using plasmid containing strains, since the hns/hfp and hns/stpA double mutants were unable to grow at $45^{\circ} \mathrm{C}$ in the presence of antibiotics in the growth medium. For the detection of siderophores, cultures were grown overnight in MM9 medium (Schwyn and Neilands, 1987) and iron-limited conditions were induced during $3 \mathrm{~h}$ by adding $0.1 \mathrm{mM}$ of the chelator $2,2^{\prime}$-dipyridyl. When required, antibiotics carbenicillin and kanamycin were used at concentrations of $100 \mu \mathrm{g} \mathrm{ml}^{-1}$ and $30 \mu \mathrm{g} \mathrm{ml}^{-1}$ respectively.

\section{Mutagenesis}

The construction of deletion mutants was performed as described earlier (Müller et al., 2006) using the $\lambda$-Red mutagenesis method (Datsenko and Wanner, 2000). The chromosomal $h f p-l a c Z$ transcriptional fusion strain was constructed in a similar approach: a $6.5 \mathrm{~kb}$ linear DNA fragment containing a promoterless lac $Z$ gene followed by lac $Y$, lac $A$ and the neo gene, promoting kanamycin resistance, was PCR amplified with primers Hfp-lacZ1 and Hfp-lacZ2, using plasmid pVIK112 as template. The primers contained $54 \mathrm{nt}$ and $48 \mathrm{nt}$ homology extensions that allowed $\lambda$-Red recombinase-mediated recombination into the intergenic region between the $h f p$ gene and the downstream ORF ECP_1928, thus leaving an intact copy of the hfp gene on the chromosome. Bacterial clones with successful integration were selected on agar plates containing 5-bromo-4-chloro-3indoyl- $\beta$-D-galactopyranoside (X-gal) and kanamycin. All mutations were confirmed by both PCR and Southern hybridization.

\section{Plasmid construction}

For overexpression of His-tagged Hfp protein, the hfp sequence was amplified from the chromosome of UPEC strain 536 by using primers Xhol-His-Hfp and Hfp-HindIII, which contained a hexa-His sequence, a factor XA cleavage site, as well as Xhol or Hindlll restriction sites. After enzymatic digest, the fragment was ligated into the $\mathrm{Xhol} / \mathrm{HindIII}$ sites of the expression plasmid pASK75, resulting in plasmid pCM8. For complementation experiments, the native $h f p$ gene with its own promoter was amplified by PCR using primers Hfp-fw-HindIII and Hfp-rv-Xbal, and the 750 bp fragment was cloned into the HindIII/Xbal sites of the low-copynumber plasmid pWKS30, resulting in plasmid pCM9. The promoter fusion constructs were created by PCR amplification of different parts of the upstream region of the hfp gene using the Hfp-EcoRI series of forward primers in combination with the reverse primer Hfp-BamHI binding immediately after the translational start codon of the $h f p$ sequence. The PCR products were cloned into the EcoRI/BamHI sites of plasmid pRZ5202, resulting in plasmids pCM15-pCM19. All inserts were sequenced for verification.

\section{RNA isolation and RT-PCR analysis}

Total RNA extraction and semi-quantitative RT-PCR were performed as described previously (Müller et al., 2006). The 
sequences of the oligonucleotides used for RT-PCR are listed in Table S3 in Supporting information.

\section{Phenotypic tests}

The utilization of beta-glucosides ( $b g /$ phenotype) was detected on agar plates containing indicator dye and $40 \mathrm{\mu g} \mathrm{ml}^{-1} \quad$ 5-Bromo-4-chloro-3-indolyl-D-glucopyranoside (X-Glu; Sigma) as substrate, where white colonies were scored as bgl-negative, and blue colonies as bgl-positive (Johansson et al., 1998). Motility, haemolytic activity and the production of siderophores were assessed as described before (Müller et al., 2006). Production of curli adhesin was detected after 3 days of incubation on no-salt LB plates supplemented with $40 \mathrm{mg} \mathrm{l}^{-1}$ congored dye and $20 \mathrm{mg} \mathrm{l}^{-1}$ Coomassie Brilliant Blue R-250. Results were scored as $+=$ red colonies or $-=$ white colonies. Auto-aggregation was assessed at room temperature in $\mathrm{M} 63 \mathrm{BI}$ medium after adjusting optical densities of the cultures as described elsewhere (Roux et al., 2005) and reported here as absent (-), slow (+) and fast $(++)$.

\section{$\beta$-Galactosidase assay}

The $\beta$-galactosidase activity was determined as described by Miller (1972). Experiments were performed in triplicate from independent cultures, with three separate measurements for each sample.

\section{Purification of recombinant His-Hfp protein}

Escherichia coli strain BL21 (DE3) $\Delta h n s$ was used for overexpression of recombinant Hfp protein from plasmid pCM8. Approximately $800 \mathrm{ml}$ of LB cultures were grown at $30^{\circ} \mathrm{C}$ to $\mathrm{OD}_{600}=0.2$, before adding the inducer anhydro-tetracycline at a final concentration of $0.2 \mu \mathrm{g} \mathrm{ml}^{-1}$. Protein synthesis was carried out for $6 \mathrm{~h}$. Cells were pelleted by centrifugation and

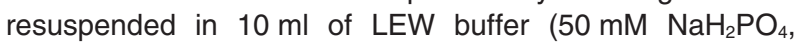
$300 \mathrm{mM} \mathrm{NaCl}, \mathrm{pH}$ 8) containing protease inhibitor cocktail (Complete Mini, Roche). Cells were lysed by sonication for $10 \times 20 \mathrm{~s}$ pulses with $20 \mathrm{~s}$ cooling periods in between. After two rounds of centrifugation at $18000 \mathrm{~g}$ for $20 \mathrm{~min}$ at $4^{\circ} \mathrm{C}$, the cleared lysate was loaded onto $\mathrm{Ni}^{2+}-$ columns (Protino Ni1000, Macherey-Nagel). Affinity chromatography was performed following the manufacturer's recommendations. Eluted protein was dialysed into storage buffer [150 mM $\mathrm{NaCl}, 50 \mathrm{mM}$ Tris- $\mathrm{HCl}$ (pH 8), 1 mM EDTA, 0.5 mM DTT, 20\% glycerol] and concentrated using Vivaspin 10000 columns (Vivascience). The His-tag was removed by factor XA digest (New England Biolabs) overnight at room temperature. For final purification by gel filtration, $1 \mathrm{ml}$ of protein samples after factor XA treatment were loaded onto a HiLoad Superdex 75 prep grade column (Amersham Biosciences) and eluted with a buffer containing $150 \mathrm{mM} \mathrm{NaCl}, 50 \mathrm{mM}$ Tris- $\mathrm{HCl}(\mathrm{pH}$ 7.5) in $2 \mathrm{ml}$ fractions.

\section{In vivo protein stability assay}

Stability of native Hfp protein expressed from pCM9 was tested as described by Johansson and Uhlin, (1999). Briefly,
$20 \mathrm{ml}$ of cultures were grown to $\mathrm{OD}_{600}=0.4$, before inhibiting protein synthesis by the addition of $100 \mu^{-1}$ spectinomycin. Samples for Western blotting were taken before and $180 \mathrm{~min}$ after the addition of spectinomycin.

\section{Electrophoretic mobility shift assay (EMSA)}

DNA-protein interactions were studied by a gel retardation assay using pure $\mathrm{H}-\mathrm{NS}$ and StpA proteins or recombinant $\mathrm{Hfp}$ protein purified from $E$. coli BL21 $\Delta h n s$ as described above. As substrate for binding, $400 \mathrm{bp}$ PCR products containing the promoter regions of $h f p, h n s$ and $\operatorname{stp} A$ were used. The composition of the reaction buffer was: $25 \mathrm{mM}$ HEPES ( $\mathrm{pH} 7.3)$, $0.1 \mathrm{mM}$ EDTA, $5 \mathrm{mM}$ DTT, $10 \%$ glycerol, $50 \mathrm{mM} \mathrm{KCl}$ and $0.1 \mu \mathrm{g}$ of poly(dldC) non-specific competitor DNA. Purified protein $(0-27 \mu \mathrm{M})$ was incubated with $100 \mathrm{ng}$ of PCR products for $20 \mathrm{~min}$ at room temperature. Samples were loaded onto $6 \%$ Tris-glycine gels and DNA was visualized by ethidium bromide staining.

\section{Chemical cross-linking}

Interactions between $\mathrm{Hfp}$ and $\mathrm{H}-\mathrm{NS}$ were studied by chemical cross-linking for $20 \mathrm{~min}$ at room temperature using $2.5 \mathrm{mM}$ glutaraldehyde as described previously (Lindberg et al., 2008). Cross-linked products were analysed by immunoblotting.

\section{Immunoblotting}

The detection of fimbrial proteins using specific antisera was performed as described previously (Müller et al., 2006). Primary antisera against H-NS or StpA were derived from immunogenized rabbits and were used in 10000 -fold dilutions. The serum originally directed against StpA strongly reacted with the Hfp protein and therefore could be used for the detection of $\mathrm{Hfp}$. Antiserum recognizing the alpha-subunit of the DNA polymerase was used as a loading control on stripped membranes as performed previously (Müller et al., 2006).

\section{Capsule ELISA}

Quantitative measurements of the production levels of the K15 capsule were performed as described (Schneider et al., 2004).

\section{In silico analyses}

Promoter regions were analysed using the bend.it and model.it servers, which are accessible at http://hydra.icgeb. trieste.it/ kristian/dna (Munteanu et al., 1998). Promoter prediction was performed using the BPROM software available at the SoftBerry Homepage (http://www.softberry.com).

\section{Statistical analyses}

Differences between average values were tested for significance by performing an unpaired, two-sided Student's $t$-test. 
The levels of significance of the resulting $P$-values are reported by the following symbols: ${ }^{*}=P<0.05,{ }^{* *}=P<0.01$, ${ }^{\star \star \star}=P<0.001$ and n.s. $=$ non-significant.

\section{Acknowledgements}

We thank Emma Andersson for her contribution to the work with the lacZ reporter strains. The Würzburg group was supported by the Deutsche Forschungsgemeinschaft (SFB479, TP A1). The work in Umeå was supported by grants from the Swedish Research Council, the Swedish Foundation for International Cooperation in Research and Higher Education (STINT), the Faculty of Medicine at Umeå University, and it was performed within the Umeå Centre for Microbial Research (UCMR). G.S. and L.E. were supported by the Hungarian Research Foundation (OTKA 62092). This work was carried out in the frame of the European Virtual Institute for Functional Genomics of Bacterial Pathogens (CEE LSHBCT-2005-512061) and the ERA-NET project 'Deciphering the intersection of commensal and extraintestinal pathogenic E. coli.

\section{References}

Atlung, T., and Ingmer, H. (1997) H-NS: a modulator of environmentally regulated gene expression. Mol Microbiol 24: 7-17.

Beloin, C., Deighan, P., Doyle, M., and Dorman, C.J. (2003) Shigella flexneri 2 a strain $2457 \mathrm{~T}$ expresses three members of the H-NS-like protein family: characterization of the Sfh protein. Mol Genet Genomics 270: 66-77.

Bertin, P., Hommais, F., Krin, E., Soutourina, O., Tendeng, C., Derzelle, S., and Danchin, A. (2001) H-NS and H-NS-like proteins in Gram-negative bacteria and their multiple role in the regulation of bacterial metabolism. Biochimie 83: 235241.

Bloch, V., Yang, Y., Margeat, E., Chavanieu, A., Auge, M.T., Robert, B., et al. (2003) The H-NS dimerization domain defines a new fold contributing to DNA recognition. Nat Struct Biol 10: 212-218.

Carniel, E. (2001) The Yersinia high-pathogenicity island: an iron-uptake island. Microbes Infect 3: 561-569.

Corbett, D., Bennett, H.J., Askar, H., Green, J., and Roberts, I.S. (2007) SlyA and H-NS regulate transcription of the Escherichia coli K5 capsule gene cluster, and expression of slyA in Escherichia coli is temperature-dependent, positively autoregulated, and independent of $\mathrm{H}-\mathrm{NS}$. J Biol Chem 282: 33326-33335.

Cusick, M.E., and Belfort, M. (1998) Domain structure and RNA annealing activity of the Escherichia coli regulatory protein StpA. Mol Microbiol 28: 847-857.

Dame, R.T. (2005) The role of nucleoid-associated proteins in the organization and compaction of bacterial chromatin. Mol Microbiol 56: 858-870.

Dame, R.T., Luijsterburg, M.S., Krin, E., Bertin, P., Wagner, R., and Wuite, G.J.L. (2005) DNA bridging: a property shared among H-NS-like proteins. J Bacteriol 187: 1845-1848.

Datsenko, K.A., and Wanner, B.L. (2000) One-step inactivation of chromosomal genes in Escherichia coli K-12 using PCR products. Proc Natl Acad Sci USA 97: 6640-6645.
Deighan, P., Beloin, C., and Dorman, C.J. (2003) Three-way interactions among the Sfh, StpA and H-NS nucleoidstructuring proteins of Shigella flexneri 2a strain 2457T. Mol Microbiol 48: 1401-1416.

Dobrindt, U., Blum-Oehler, G., Hartsch, T., Gottschalk, G., Ron, E.Z., Fünfstück, R., and Hacker, J. (2001) S-fimbriaencoding determinant sfal is located on pathogenicity island $\mathrm{III}_{536}$ of uropathogenic Escherichia coli strain 536. Infect Immun 69: 4248-4256.

Dorman, C.J. (2004) H-NS: a universal regulator for a dynamic genome. Nat Rev Microbiol 2: 391-400.

Doyle, M., and Dorman, C.J. (2006) Reciprocal transcriptional and posttranscriptional growth-phase-dependent expression of $s f h$, a gene that encodes a paralogue of the nucleoid-associated protein H-NS. J Bacteriol 188: 75817591.

Doyle, M., Fookes, M., Ivens, A., Mangan, M.W., Wain, J., and Dorman, C.J. (2007) An H-NS-like stealth protein aids horizontal DNA transmission in bacteria. Science 315 : 251-252.

Free, A., and Dorman, C.J. (1997) The Escherichia coli stpA gene is transiently expressed during growth in rich medium and is induced in minimal medium and by stress conditions. J Bacteriol 179: 909-918.

Göransson, M., Sonden, B., Nilsson, P., Dagberg, B., Forsman, K., Emanuelsson, K., and Uhlin, B.E. (1990) Transcriptional silencing and thermoregulation of gene expression in Escherichia coli. Nature 344: 682-685.

Hommais, F., Krin, E., Laurent-Winter, C., Soutourina, O., Malpertuy, A., Le Caer, J.P., et al. (2001) Large-scale monitoring of pleiotropic regulation of gene expression by the prokaryotic nucleoid-associated protein, H-NS. Mol Microbiol 40: 20-36.

Johansson, J., and Uhlin, B.E. (1999) Differential proteasemediated turnover of $\mathrm{H}-\mathrm{NS}$ and StpA revealed by a mutation altering protein stability and stationary-phase survival of Escherichia coli. Proc Natl Acad Sci USA 96: 1077610781.

Johansson, J., Dagberg, B., Richet, E., and Uhlin, B.E. (1998) H-NS and StpA proteins stimulate expression of the maltose regulon in Escherichia coli. J Bacteriol 180: 61176125.

Johansson, J., Eriksson, S., Sondén, B., Wai, S.N., and Uhlin, B.E. (2001) Heteromeric interactions among nucleoid-associated bacterial proteins: localization of StpA-stabilizing regions in $\mathrm{H}-\mathrm{NS}$ of Escherichia coli. J Bacteriol 183: 2343-2347.

Lang, B., Blot, N., Bouffartigues, E., Buckle, M., Geertz, M., Gualerzi, C.O., et al. (2007) High-affinity DNA binding sites for $\mathrm{H}-\mathrm{NS}$ provide a molecular basis for selective silencing within proteobacterial genomes. Nucleic Acids Res 35: 6330-6337.

Lindberg, S., Xia, Y., Sondén, B., Göransson, M., Hacker, J., and Uhlin, B.E. (2008) Regulatory interactions among adhesin gene systems of uropathogenic Escherichia coli. Infect Immun 76: 771-780.

Lithgow, J.K., Haider, F., Roberts, I.S., and Green, J. (2007) Alternate SlyA and H-NS nucleoprotein complexes control hlyE expression in Escherichia coli K-12. Mol Microbiol 66: 685-698.

Luijsterburg, M.S., Noom, M.C., Wuite, G.J.L., and Dame, 
R.T. (2006) The architectural role of nucleoid-associated proteins in the organization of bacterial chromatin: a molecular perspective. J Struct Biol 156: 262-272.

Madrid, C., Balsalobre, C., Garcia, J., and Juarez, A. (2007) The novel Hha/YmoA family of nucleoid-associated proteins: use of structural mimicry to modulate the activity of the H-NS family of proteins. Mol Microbiol 63: 7-14.

Mayer, O., Rajkowitsch, L., Lorenz, C., Konrat, R., and Schroeder, R. (2007) RNA chaperone activity and RNAbinding properties of the E. coli protein StpA. Nucleic Acids Res 35: 1257-1269.

Miller, J. (1972) Experiments in Molecular Genetics. Cold Spring Harbor, NY: Cold Spring Harbor Laboratory Press.

Müller, C.M., Dobrindt, U., Nagy, G., Emödy, L., Uhlin, B.E., and Hacker, J. (2006) Role of histone-like proteins H-NS and StpA in expression of virulence determinants of uropathogenic Escherichia coli. J Bacteriol 188: 5428-5438.

Munteanu, M.G., Vlahovicek, K., Parthasarathy, S., Simon, I., and Pongor, S. (1998) Rod models of DNA: sequencedependent anisotropic elastic modelling of local bending phenomena. Trends Biochem Sci 23: 341-347.

Owen-Hughes, T.A., Pavitt, G.D., Santos, D.S., Sidebotham, J.M., Hulton, C.S.J., Hinton, J.C.D., and Higgins, C.F. (1992) The chromatin-associated protein H-NS interacts with curved DNA to influence DNA topology and gene expression. Cell 71: 255-265.

Renzoni, D., Esposito, D., Pfuhl, M., Hinton, J.C.D., Higgins, C.F., Driscoll, P.C., and Ladbury, J.E. (2001) Structural characterization of the $\mathrm{N}$-terminal oligomerization domain of the bacterial chromatin-structuring protein, H-NS. J Mol Biol 306: 1127-1137.

Rimsky, S., Zuber, F., Buckle, M., and Buc, H. (2001) A molecular mechanism for the repression of transcription by the H-NS protein. Mol Microbiol 42: 1311-1323.

Roux, A., Beloin, C., and Ghigo, J.-M. (2005) Combined inactivation and expression strategy to study gene function under physiological conditions: application to identification of new Escherichia coli adhesins. J Bacteriol 187: 10011013.

Schneider, G., Dobrindt, U., Brüggemann, H., Nagy, G., Janke, B., Blum-Oehler, G., et al. (2004) The pathogenicity island-associated $\mathrm{K} 15$ capsule determinant exhibits a novel genetic structure and correlates with virulence in uropathogenic Escherichia coli strain 536. Infect Immun 72: 5993-6001.

Schwyn, B., and Neilands, J.B. (1987) Universal chemical assay for the detection and determination of siderophores. Anal Biochem 160: 47-56.

Sondén, B., and Uhlin, B.E. (1996) Coordinated and differential expression of histone-like proteins in Escherichia coli: regulation and function of the H-NS analog StpA. EMBO $J$ 15: 4970-4980.

Sonnenfield, J.M., Burns, C.M., Higgins, C.F., and Hinton, J.C. (2001) The nucleoid-associated protein StpA binds curved DNA, has a greater DNA-binding affinity than $\mathrm{H}-\mathrm{NS}$ and is present in significant levels in hns mutants. Biochimie 83: 243-249.

Tendeng, C., and Bertin, P. (2003) H-NS in Gram-negative bacteria: a family of multifaceted proteins. Trends Microbiol 11: 511-518.

Travers, A., and Muskhelishvili, G. (2005) Bacterial chromatin. Curr Opin Genet Dev 15: 507-514.

Ueguchi, C., Seto, C., Suzuki, T., and Mizuno, T. (1997) Clarification of the dimerization domain and its functional significance for the Escherichia coli nucleoid protein $\mathrm{H}-\mathrm{NS}$. $J$ Mol Biol 274: 145-151.

Westermark, M., Oscarsson, J., Mizunoe, Y., Urbonaviciene, J., and Uhlin, B.E. (2000) Silencing and activation of ClyA cytotoxin expression in Escherichia coli. J Bacteriol 182: 6347-6357.

Williams, R.M., and Rimsky, S. (1997) Molecular aspects of the $E$. coli nucleoid protein, H-NS: a central controller of gene regulatory networks. FEMS Microbiol Lett 156: 175185.

Yamada, H., Muramatsu, S., and Mizuno, T. (1990) An Escherichia coli protein that preferentially binds to sharply curved DNA. J Biochem 108: 420-425.

Zhang, A., Rimsky, S., Reaban, M.E., Buc, H., and Belfort, M. (1996) Escherichia coli protein analogs StpA and H-NS: regulatory loops, similar and disparate effects on nucleic acid dynamics. EMBO J 15: 1340-1349.

\section{Supporting information}

Additional supporting information may be found in the online version of this article.

Please note: Wiley-Blackwell are not responsible for the content or functionality of any supporting materials supplied by the authors. Any queries (other than missing material) should be directed to the corresponding author for the article. 